\title{
Enhancing Wireless Video Transmissions in Virtual Collaboration Environments
}

\author{
O. Abdul-Hameed, ${ }^{[1]}$ S. Nasir, ${ }^{[1]}$ H. Karim, ${ }^{[1]}$ T. Masterton, ${ }^{[2]}$ and A. M. Kondoz ${ }^{[1]}$
}

\begin{abstract}
This paper introduces the virtual collaboration environment and discusses the problems encountered in wireless video transmissions of the participating users. Different schemes are proposed and evaluated to address various problems encountered in the wireless access links of the virtual collaboration system for enhancing the perceived visual quality. The schemes include radio network resource optimization, optimal joint source and channel rate allocation and error resilience enhancement using SVC-MDC. These schemes have been shown to offer a strong potential to be incorporated in a virtual collaboration system for quality enhancement.
\end{abstract}

Index Terms-Heterogeneous environment, rate allocation, SVC-MDC, virtual collaboration system.

\section{INTRODUCTION}

$\mathrm{T}$ HE virtual collaboration environment is a system where remotely located users meet in a virtual environment created by the audiovisual technologies as if they were located in the same room. In this paper, we focus on QoS provisioning in the wireless segments of the virtual collaboration environment using the Virtual Collaboration System (VCS) described by VISNET II Network of Excellence (NoE) of the IST FP6 programme. The VCS is currently being used and improved by the NoE partners for achieving cross theme integration by bringing together the work performed in the three VISNET II themes, being video coding, audiovisual media processing and security.

Collaborative Working Environments (CWEs) are identified either as "synchronous" where both parties communicate in real time to achieve a common goal or "asynchronous" where they share common artefacts in a workspace but users may access them individually at different times as defined by workflow considerations. Real time audio and video technologies are required for the basic video conferencing element of synchronous collaboration. Means for contact management, link establishment, communications security,

[1] O. Abdul-Hameed, S. Nasir, H. Karim and A. M. Kondoz are with the I-Lab/Centre for Communication Systems Research (CCSR), University of Surrey, Guildford GU2 7XH, UK (Tel: +44 (0) 1483 686002; Fax: +44 (0) 1483 686011; emails: \{O.Abdul-Hameed, S.Nasir, H.Karim A.Kondoz\}@surrey.ac.uk).

[2] T. Masterton is with Thales Research and Technology (UK) Ltd., Worton Drive, Worton Grange Business Park, Reading RG2 0SB, Berkshire, UK (Tel: +44 (0) 1189238266; Fax: +44 (0) 1189238399; email: tim.masterton@thalesgroup.com). access control and link encryption are also required. Internet Protocol (IP) is the connection infrastructure of choice as it supports all required types of communication and is becoming ever more widespread with high capacity. However it brings the limitation of occasional unpredictability, particularly for delays in real time audio and video streams. Investigation of the connectivity problems that occur and Quality of Service (QoS) technology to ensure that the user perception is not unduly affected by link imperfections is necessary. Asynchronous collaboration adds the requirements for a persistent store of information that is not dependent on the availability of any particular user or terminal and meets certain availability criteria. It also requires the presence of work flow elements in the system for controlling access sequences, handling the human factors issues of notifying changes by other users, maintaining efficiency, controlling release states and achieving transactions on the workspace.

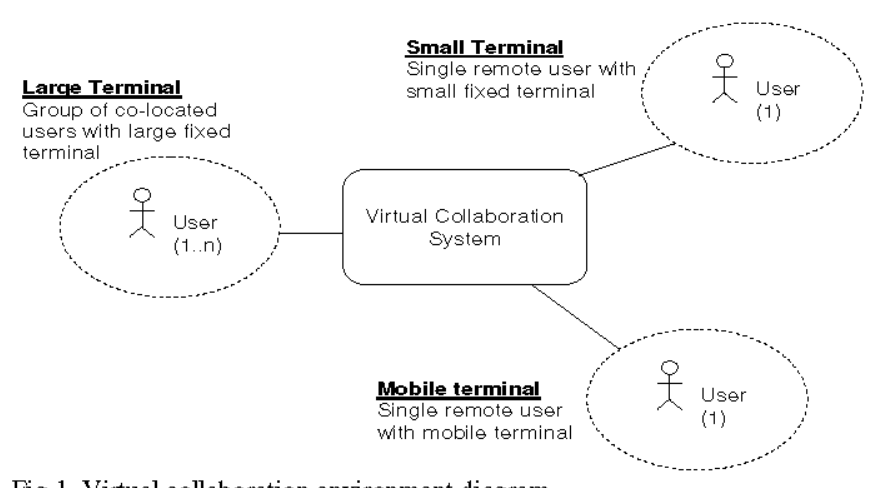

Fig.1. Virtual collaboration environment diagram.

The rest of the paper is organized as follows: Section II discusses the problems encountered in the wireless segment of a virtual collaboration environment. In Section III, we propose solutions to these problems. Section IV concludes the paper.

\section{PROBLEM STATEMENT}

\section{A. Heterogeneous Access Networks}

In a virtual collaboration environment, remote users may participate in a virtual collaboration session using different access links and devices. The access links can be wired or wireless. Considering wireless access links, different options are available, for example GSM, GPRS, UMTS, WLAN or WiMAX [13]. In video transmissions over these heterogeneous wireless access technologies, problems may 
arise due to the differences in available data rates (for example, up to $2 \mathrm{Mbps}$ in UMTS, up to $54 \mathrm{Mbps}$ in WLAN and up to $70 \mathrm{Mbps}$ in WiMAX), QoS discrepancies and timevarying transmission characteristics of the wireless channels due to user mobility and interference [14]. Therefore, it is necessary to setup the required resources so that the expected end to end QoS may be achieved by negotiating suitable traffic and QoS parameters and optimizing the radio network resources accordingly. In addition, it is also necessary to have the dynamic adaptation mechanisms that take into account the time-varying characteristics of the wireless channel.

\section{B. Source Channel Rate Allocation Optimisation}

Prioritised content adaptation can be applied for the efficient use of radio network resources under specific network conditions such as network overload situations. Certain regions of the input video bit stream can be assigned more importance as compared to other regions of the video frame that may be of less interest. A particular user for example who is involved in a videoconference using a mobile device may be more interested in the talking person's region as compared to other participants. Similarly, viewers would be more interested in the action area of a particular news clip being aired on a mobile TV application. This can be achieved by prioritising different parts of the video bit stream and sending them using multiple radio bearers with different characteristics, such as channel coding, modulation etc. This would essentially require optimally separating the encoded bit stream into a number of sub-streams and allocating source and channel resources to each stream according to the perceived importance of the particular region. Joint source-channel coding approaches [1] have proven to significantly enhance performances for video applications over practical systems. Application scenarios implementing these techniques have been discussed in $[2,6]$ that closely relate to the Virtual collaboration scenario.

\section{Scalability Issues}

If users with various different contexts (including location, terminal capability, and link parameters) wish to collaborate as well as possible using with their available access links, the overall collaboration system will be heterogeneous and so elements must be placed in the network to scale content appropriately for a range of device capabilities. For example some specialist workers may need to have their hands free while collaborating, whereas others may have the luxury of presentation quality interfaces and vast quantities of information to analyse. This requires context detection, stream adaptation and scalable encoding for video, audio and data streams. The scalable encoding for video data issue in VCS shall be well addressed by a well known technique in video coding community, namely Scalable Video Coding (SVC). It allows the decoding of appropriate subsets of bit-stream to generate complete pictures of size and quality dependent on the proportion of the total bit stream decoded. Universal media access in virtual collaboration system can be made possible with SVC by coding the video only once to achieve a scalable coded stream. This stream can then be accessed 'anytime', from 'anywhere' using any access network such as wireless and Internet, and by any terminal complexity. The scalable properties come at the expense of decreased coding efficiency as it often introduces a degree of data redundancy. In general, SVC can be divided into three basic types namely quality scalability (Signal to Noise Ratio (SNR) scalability), spatial scalability and temporal scalability. Combination of three scalable types can also be achieved to produce hybrid scalability [8]. SVC produces video layers which consist of a base layer and a number of enhancement layers. The base layer is essential for the video decoder to produce a basic acceptable output. The enhancement layers help to improve the visual quality if received by the decoder. Without the base layer, enhancement layers only are useless to the decoder. The video quality issue in virtual collaboration system is partly caused by a packet loss problem in the main Internet. Hence, there are two issues that should be addressed in the VCS being error resilience and scalability aspect of the video. One of the ways to address these problems is by using scalable multiple description coding (SVC-MDC) to encode the video. SVC$\mathrm{MDC}$ has been proposed to improve error resilience of the transmitted video over unreliable networks and at the same time provide adaptation to bandwidth variations and receiving device characteristics [9].

\section{PROPOSED SOLUTIONS}

\section{A. Setting up the Required Resources for Achieving the Expected End to End QoS}

Considering a scenario where an emergency personnel user requiring to participate in a crisis management virtual collaboration session using a handheld terminal while being in a WCDMA UMTS coverage area. If at the time of the connection request the UMTS cell was overloaded, the user may be denied access to the network [12] and hence to the virtual collaboration session. A scheme proposed in [15] was modified to produce a scheme for optimizing the radio network resources based on users' QoS parameters (e.g. bit rate, blocking and dropping probabilities) and user's preferences (e.g. video resolution, error resilience) for improving the blocking probability for high priority incoming call requests that are classified into three levels being Enhanced, Medium and Normal according to how much the users are paying for the service. Users belonging to the Enhanced level such as an emergency personnel user will have an enhanced QoS, whereas users belonging to the Medium level will have moderate QoS and users belonging to the Normal level will have normal QoS in terms of blocking probability. The scheme is illustrated in the following flowchart in Fig. 2. The scheme's performance was evaluated using an implemented and validated with [12] system level simulator for WCDMA UMTS. Fig. 3 shows the accepted traffic versus the offered traffic curves in erlangs/cell. The top two red curves are for voice traffic at $12.2 \mathrm{kbps}$ for validation. The bottom three curves are for video with the top curve for 
video at $64 \mathrm{kbps}$, the bottom curve for video at $128 \mathrm{kbps}$, whereas the middle curve is obtained when using the scheme which indicates improved accepted traffic (or equivalently lower blocking probability) for the Enhanced video users.

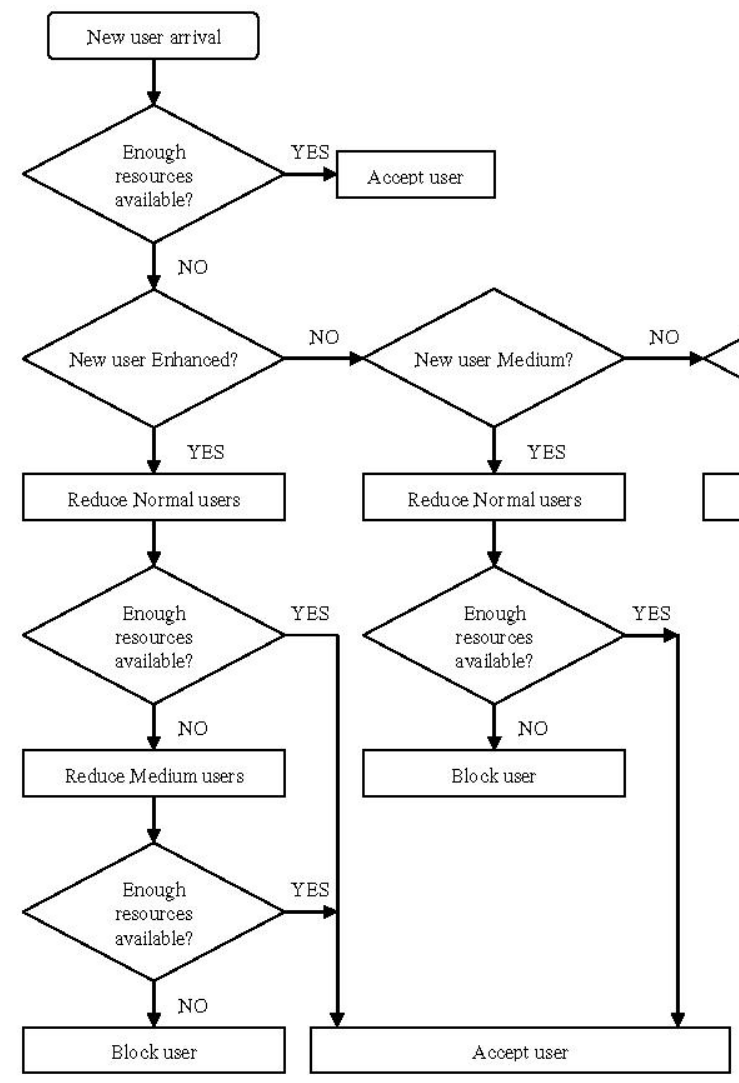

Fig. 2. Flow chart illustrating the radio network resource optimisation scheme.

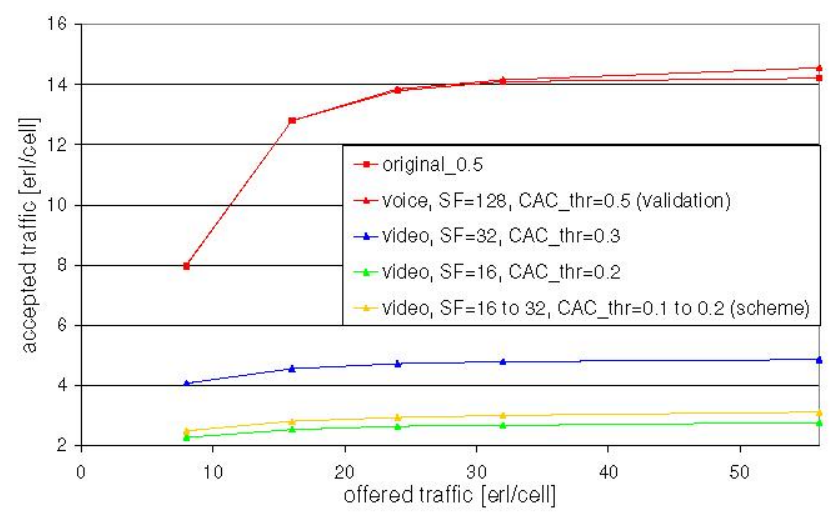

Fig. 3. Accepted traffic versus offered traffic for different rates.

\section{B. Optimal Joint Source Channel Bit Rate Allocation}

In this subsection, we introduce the design of an optimal joint source channel bit rate allocation for video transmissions over wireless networks. The proposed scheme utilizes bit level unequal error protection (UEP) and joint source channel coding to obtain optimal video quality over a wide range of channel conditions. The encoded video data is separated into a number of sub-streams based on the relative importance of data in different video packets, which is calculated by the estimated perceived importance of bits at the encoder. It then applies differential protection by sending prioritised data over multiple radio bearers. Realisation of the proposed scheme is presented in Fig. 4 below.

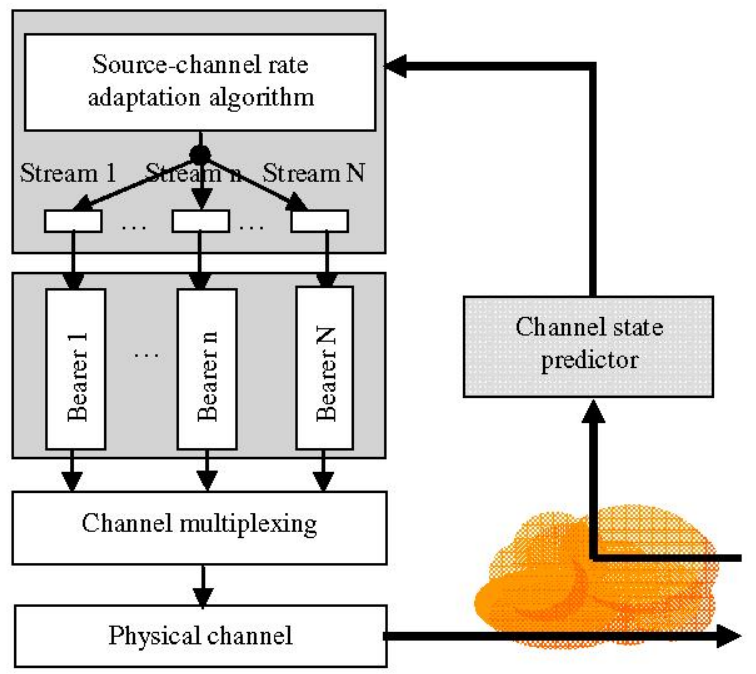

Fig. 4. Realisation of proposed rate allocation scheme.

The transmission channel is characterised by the probability of channel bit errors and the channel bandwidth, $R_{c k}$, expressed in terms of bits per second. Assume that the underlying communication system allows a maximum of $N$ communication sub-channels or radio bearers for a given video service. The encoded bit stream is separated into $N$ number of sub-streams. $R_{n}$, denotes the channel bit rate on the $n^{\text {th }}$ subchannel, and $\chi_{n}$ is the channel coding rate on the $n^{\text {th }}$ subchannel. The optimum possible source rate, $R$, is a function of channel bit rate, $R_{n}$, and channel coding rate, $\chi_{n}$

$$
\begin{aligned}
& R=R_{1}+\cdots+R_{n}+\cdots+R_{N} \\
& R_{c h} \geq R_{1} / \chi_{1}+\cdots+R_{n} / \chi_{n}+\cdots+R_{N} / \chi_{N}
\end{aligned}
$$

The expected distortion due to the corruption of the $n^{\text {th }}$ substream is $E\left(D_{n}\right)$. Hence the total sequence distortion $E(D)$ becomes:

$$
E(D)=\sum_{n=1}^{N} E\left(D_{n}\right)
$$

The goal set here is to find the optimal sub-stream separation and mapping of sub-stream data onto multiple radio bearers in such a way to maximise the received video quality for video transmission over a bandwidth limited error prone wireless channel. The optimisation problem can formally be written as:

Minimise $\quad E(D)=\sum_{n=1}^{N} E\left(D_{n}\right)$

Subject to:

$R_{1} / \chi_{1}+\cdots+R_{n} / \chi_{n}+\cdots+R_{N} / \chi_{N} \leq R_{c h}$

The proposed algorithm operates at video frame level with the estimation of the source rate, $R$, for given channel 
bandwidth/protection. After encoding the video frame with the estimated source rate, the expected distortion is calculated at video packet level. Based on the calculated distortion values, data partition of each video packet is assigned an importance level. Data separation into $N$ number of sub-streams is performed based on the calculated importance levels. Instantaneous channel quality for each selected sub-channel is predicted. The source rates for sub-channels are calculated and the channel bandwidth requirement set out in Equation 4 is checked. If the bandwidth requirement is not satisfied, the data on the highly protected sub-channels are reduced and the importance levels are re-calculated. Expected frame distortion is calculated again and the step is repeated until the minimum distortion value is obtained.

The distortion for current frame being encoded is estimated using the model presented in [5]. Standard object segmentation available in MPEG-4 is used for these experiments. The "Singer" and "Kettle" test sequences have been used in this experiment. The "Singer" sequence is used as the background and the "Kettle" sequence is segmented and used in the foreground of the output sequence each transmitted over different radio bearers with unequal error protection. The CIF ( $352 \times 288$ pixels) sequences are coded at $30 \mathrm{fps}$.

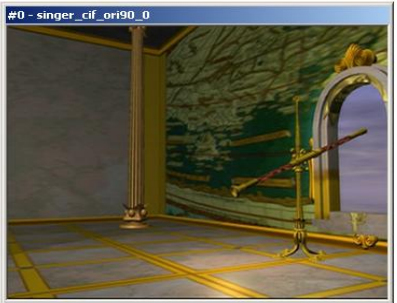

(a)

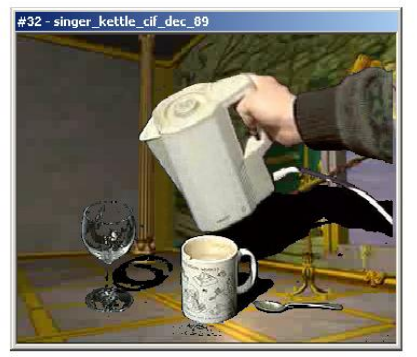

(c)

Fig. 5. Input sequences: (a) Singer.cif, (b) Kettle.cif, (c) Output composite image at decoder.

\section{Error Resilience Enhancement Using SVC-MDC}

SVC-MDC aims to combine the flexibility of scalable coding with the robustness of MDC. The objective of the SVC-MDC scheme is to enhance error resilience of the base layer of SVC using temporal MDC. The base layer is the most important part of the SVC bitstream, as all the other layers depend on it. Therefore, it is important to make it error robust. The temporal MDC of the base layer is produced using the multi-state video coding approach as in [10] which separates the even and odd frames of a sequence into two MDC streams as shown in Fig. 6. Streams 1 and 2 contains even and odd frames respectively. With this approach, MDC loses its coding efficiency due to the prediction from previous two frames and longer motion vector need to be coded.
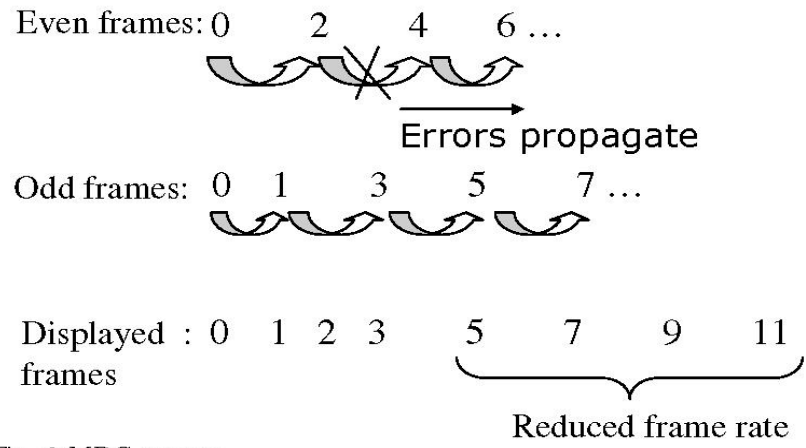

Fig. 6. MDC streams.

If both the even and the odd streams are received, the decoder can reconstruct the coded sequence at full temporal resolution. If only one of the streams is received, the decoder can still decode the received stream at half the original temporal resolution. Since the even frames are predicted from previous even frames (independent from odd frames), there will be no mismatch if one of the streams are lost at the decoder. Additionally, in the case of one stream being received, the decoder can decode at the full resolution by interpolating between the received frames as in [10] or by repeating the frame. If error occurs in one of the frames in the MDC stream (frame 4) as shown in Fig. 6, the error will propagate to next even frame. The MDC decoder can then switch to the odd stream and display the frame at reduced frame rate. Alternatively, the MDC decoder can interpolate between the reduced frame rates or repeat the frame to achieve full temporal resolution. The Joint Scalable Video Model (JSVM) software has been modified to produce the even and odd frame MDC scheme. The preliminary subjective result for error prone is shown in Fig. 7 to simulate VCS access using wired Internet. 20\% packet loss of Internet error pattern is used [11]. Interview sequence at CIF resolution and $30 \mathrm{fps}$ is coded using the original JSVM software (SDC) and modified JSVM software (MDC) to achieve the same bit rate of about $300 \mathrm{kbps}$ by varying the quantisation parameter. Table 1 shows the quantisation parameter and the I-frame rate used to achieve the bit rate. In order to achieve the same bit rate as MDC at the same quantisation parameter, SDC used more Iframes than MDC.

TABLE I

PARAMETERS USED FOR THE SDC AND MDC ENCODER

\begin{tabular}{|l|l|l|c|c|}
\hline Coder & $\begin{array}{l}\mathrm{Q} \\
\mathrm{P}\end{array}$ & I-frame rate & $\begin{array}{l}\text { Bit rate } \\
(\mathrm{kbps})\end{array}$ & $\begin{array}{l}\text { Average } \\
\text { PSNR }\end{array}$ \\
\hline SDC & 30 & Every 5 frames & 290.89 & 35.64 \\
\hline MDC & 30 & $\begin{array}{l}\text { Every 30 frames } \\
\text { in each stream }\end{array}$ & 292.85 & 35.63 \\
\hline
\end{tabular}

The 20\% packet loss is only in the even stream for MDC to simulate ideal MDC channel (one stream is completely lost), 
while in SDC, any frames can be subjected to the $20 \%$ packet loss. It is assumed that one lost corresponding of one frame lost. Fig. 7 shows the subjective results for frame 80 and comparison between interpolate and repeat frame method of the reduced frame rate. In SDC, frame copy error concealment is used. In the MDC simulation, if an error occurs in even frame, the decoder switch to odd frame and ignores the rest of the even frame. It then interpolates or repeats the odd frames to achieve full resolution. When playing the sequence, a little bit of jerky motion is observed for MDC-repeat due to the frame repetition. It can be concluded that under ideal MDC channel, MDC is very effective in combating transmission errors compared to SDC.

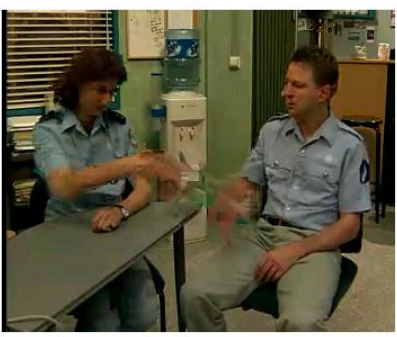

(a)

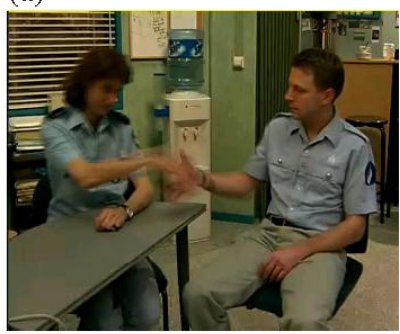

(c)

Fig. 7. Subjective results for frame number 80 of Interview sequence, (a) SDC, (b) MDC-repeat, (c) MDC-interpolate when subjected to $20 \%$ packet loss.

\section{CONCLUSION}

We have introduced the virtual collaboration environment and the problems associated with the use of wireless access links by users participating in a virtual collaboration session.

For achieving the expected end to end QoS, a radio network resource optimisation scheme based on users' QoS and preferences for enhancing the quality of high priority users who require to participate in a virtual collaboration session was evaluated and it was shown to improve the accepted traffic especially for high priority users.

For source channel rate optimization, a joint source-channel rate allocation based adaptation scheme was proposed. The rate allocation is based on the modelling of expected distortion at the video packet level. The video data can then be prioritised according to the relative perceived importance. Prioritised streams are then transmitted over the air interface by using multiple radio bearers with different error protection capabilities.

For scalability issues, scalable MDC is a promising approach to mitigate the effect of channel errors in error prone transmissions. It can provide error resilience to the base layer of SVC and at the same time provides scalability features. As for future work, more extensive tests are needed, particularly for the case if packets are lost in both of the MDC streams. Scalable features of the scalable MDC will also be investigated. Moreover, the scalable MDC will be experimented with stereoscopic video sequence for 3D virtual collaboration applications.

\section{ACKNOWLEDGEMENTS}

The work presented was developed within VISNET II, a European Network of Excellence (http://www.visnet-noe.org), funded under the European Commission IST FP6 programme. Authors would like to thank Dr Huseyin Hacihabiboglu of ILab/CCSR, University of Surrey for his review comments.

\section{REFERENCES}

[1] Bystrom. M., Stockhammer. T., "Dependent source and channel rate allocation for video transmission", IEEE Trans. Wireless Comm., Vol.3, No. 1, Jan.2004, pp 258-268

[2] Cherriman. P., Kuan. E.-L., Hanzo. L., "Burst-by-burst adaptive joint detection CDMA/H.263 based video telephony", IEEE Trans. Circuits and Systems for Video Tech., Vol.12, No.5, May 2002, pp 342-348

[3] Dyck. R.E.,Miller. D.J., "Transport of wireless video using separate, concatenated, and joint source-channel coding", Proc. IEEE, Vol. 87, No. 10, Oct. 1999 , pp 1734-1750.

[4] Gharavi, H., Alamouti, S.M., "Video transmission for third generation wireless communication systems", Journal of Research of the National Institute of Standards and Technology, Vol. 106, March-April, 2001, pp 455469.

[5] Il-Min Kim; Hyung-Myung Kim; "An optimum power management scheme for wireless video service in CDMA systems", IEEE Trans. on Wireless Comm., Vol. 2, No. 1, Jan. 2003, pp: 81-91.

[6] Kodikara, C., Fabri. S.N., Kondoz. A.M., "Link adaptation for realtime video communications in E-GPRS networks", IEE Proc. Comm., Vol. 151, No. 5, Oct. 2004, pp 438-444.

[7] Kodikara, C., Worrall, S., Fabri, S., Kondoz, A., "Performance evaluation of MPEG-4 video telephony over UMTS", in Proceedings of 3G2003, 25-27 June 2003, London, UK, pp. 73-77.

[8] H. Schwarz, D. Marpe and T. Wiegand, "Overview of the scalable H.264/MPEG4-AVC extension", in Proceedings of International Conference on Image Processing (ICIP 2006), Atlanta, GA, USA, Oct. 8-11 2006.

[9] M. v. d. Schaar and D. S. Turaga, "Multiple description scalable coding using wavelet-based motion compensated temporal filtering", in Proceedings of International Conference in Image Processing (ICIP 2003), 14-17 Sept. 2003, pp. III-489-III-492.

[10] J. G. Apostolopoulos, "Error-resilient video compression via multiple state streams", in Proceedings of International Workshop on Very Low Bitrate Video Coding, VLBV99, Kyoto, Japan, October 1999.

[11] S. Wenger, "Error patterns for Internet experiments", ITU Telecommunications Standardization Sector, Doc. Q15-I-16r1, Oct. 1999.

[12] A. Capone, S. Redana, "Call Admission Control Techniques for UMTS", in Proc. of IEEE VTC 2001 Fall, Atlantic City, NJ, Oct 2001.

[13] Shahbaz Khan, Shoaib Khan, Sahibzada Ali Mahmud, Hamed AlRaweshidy, "Supplementary Interworking Architecture for Hybrid Data Networks (UMTS-WiMAX)", in Proc. Int. Multi-Conf. on Computing in the Global Info. Technol. (ICCGI'06), Aug. 2006, pp 57-57.

[14] Harri Holma, Antti Toskala, WCDMA for UMTS Radio Access for Third Generation Mobile Communications, John Wiley \& Sons Ltd., 2000.

[15] Sund-Eun Kim, Heechang Kim, John A. Copeland, "Dynamic Radio Resource Allocation Considering QoS in UMTS Network”, IEEE 2002. 